\title{
Bickerstaff brainstem encephalitis
}

INSERM

\section{Source}

INSERM. (1999). Orphanet: an online rare disease and orphan drug data base. Bickerstaff brainstem encephalitis. ORPHA:79138

Bickerstaff's brainstem encephalitis (BBE) is a rare post-infectious neurological disease characterized by the association of external ophthalmoplegia, ataxia, lower limb arreflexia, extensor plantar response and disturbance of consciousness (drowsiness, stupor or coma). 